\title{
Two integrating spheres with an intervening scattering sample
}

\author{
John W. Pickering, Christian J. M. Moes, ${ }^{*}$ H. J. C. M. Sterenborg, Scott A. Prahl, ${ }^{\dagger}$ and Martin J. C. van Gemert \\ Laser Centre, Academic Medical Centre, Meibergdreef 9, 1105 AZ, Amsterdam, The Netherlands
}

Received March 11, 1991; revised manuscript received October 23, 1991; accepted October 25, 1991

\begin{abstract}
Two integrating spheres placed so that the exit port of one and the entry port of the other are adjacent, with only a sample intervening, will permit the simultaneous determination of the reflectance and the transmittance of the sample. Such a geometry permits measurements to be made as the sample undergoes some external stimulation, such as heat, pressure, or a chemical change. To determine the sample reflectance and the transmittance from the measured values of irradiance within each sphere requires the calculation of the exchange of light through the sample between the spheres. First the power collected by a detector situated in the wall of an integrating sphere is calculated as a function of the area and the reflectance of the wall, the holes, the sample, and the detector for both diffuse and collimated light incident upon the sample and for a sample located at either the exit port (reflectance) or the entry port (transmittance) of the sphere. Next, by using the single-sphere equations, we calculate the effect of the multiple exchange of light between two integrating spheres arranged so that the sample is placed between them. In all the cases of two integrating spheres the power detected is greater than or equal to that for the single sphere and depends on both the reflection and the transmission properties of the sample. Additionally, the effect of a baffle placed between the sample and the detector or of a nonisotropic detector is to reduce the power detected.
\end{abstract}

\section{INTRODUCTION}

Use of an integrating sphere as a means to measure the reflectance or the transmittance of a sample is an established technique. Calculations have been made that give the power detected or the radiance of the sphere as a function of the geometry of the sphere (wall area, hole area, sample area), the reflectance of the sphere, and the reflectance of the sample. ${ }^{1-5}$

Usually the sample is placed at an exit port of the sphere and is illuminated directly. Another measurement may be made by first illuminating the sphere wall or a standard placed at another port within the sphere wall. The transmittance of a sample is also measurable with the integrating sphere by placing the sample at the port of entrance to the sphere.

Our particular interest is the optics of biological tissue, with dosimetry in laser-assisted medicine and surgery being the end product of our investigations. The measurement of the total reflectance, the total transmittance, and the thickness of a sample permits the determination of the absorbing and the scattering properties of tissue under the assumption of some model of radiative transfer. ${ }^{6,7}$ The most accurate model for the slab geometry used in integrating-sphere measurements appears to be the adding-doubling method, ${ }^{8,9}$ which, with the addition of a collimated transmission measurement, permits the determination of the absorption coefficient, the scattering coefficient, and the average cosine of the scattering angle. With these three parameters other radiative transfer models more suitable to the geometries involved in laser surgery, such as Monte Carlo simulations, permit the determination of the light distribution within biological tissue during laser surgery. ${ }^{10}$

Since a temperature rise is the initial response of all tissue to laser light it is desirable to know the change in optical properties as a function of the temperature. This requires the simultaneous measurement of reflectance and transmittance (preferably both diffuse and collimated transmittance) as the tissue is being heated. This is achievable through the placement of the sample between two integrating spheres.

Such an arrangement of the spheres will permit some of the light that is initially reflected from the sample to be transmitted back through the sample and add to the signal in the second sphere (measuring transmittance). Furthermore, some of the transmitted light will be retransmitted back to the first sphere (measuring reflection), thus adding to the signal in the first sphere. This exchange of light will continue until all the light is absorbed by the sample, the walls, or the detector or is lost through the holes.

Not only are the double integrating spheres required for the simultaneous measurement of reflectance and transmittance during the heating of biological tissue but they have also been used purely for convenience. ${ }^{11,12}$ Unfortunately, neither Ref. 11 nor Ref. 12 appeared to take into account the multiple exchange of light between the spheres. Furthermore, this technique of communicating spheres may conceivably be used in many other areas of optical measurements, particularly when a sample is undergoing changes resulting from, for example, heat or chemical reactions.

Therefore, the purpose of this paper is to determine the power collected by detectors in the sphere walls as a function of the spheres' geometric and reflection parameters and the reflectance and the transmittance of the sample in the double-integrating-sphere system. Initially we calculate the detected power for a single sphere with diffuse light incident upon the sample (Section 2). From this calculation we are able to deduce the equations for collimated incident light and for transmittance measurements. The 
Table 1. Nomenclature

\begin{tabular}{cl}
\hline Nomenclature & \multicolumn{1}{c}{ Definition } \\
\hline$P$ & Power of the light entering the sphere \\
$P_{d}$ & Power collected by the detector \\
$m$ & Reflection factor of the sphere wall \\
$r$ & Reflection factor of the detector \\
$R_{d}$ & Diffuse reflection factor of the sample with diffuse incident light \\
$R_{c}$ & Collimated (specular) reflection factor of the sample with collimated incident light \\
$R_{c d}$ & Diffuse reflection factor of the sample with collimated incident light \\
$T_{d}$ & Diffuse transmission factor of the sample with diffuse incident light \\
$T_{c}$ & Collimated transmission factor of the sample with collimated incident light \\
$T_{c d}$ & Diffuse transmission factor of the sample with collimated incident light \\
$\mathfrak{N}$ & Radius of the sphere \\
$A=4 \pi \Re^{2}$ & Total sphere area \\
$\alpha A$ & Area of the sphere wall \\
$\delta$ & Area of the detector \\
$s$ & Area of the sample \\
$h$ & Area of the sphere wall relative to the total sphere area \\
$\alpha=1-(\delta / A+s / A+h / A)$ & Area of the holes \\
\hline
\end{tabular}

${ }^{a}$ When a second sphere is introduced the properties of this sphere are labeled with a prime (e.g., $\left.m^{\prime}, A^{\prime}\right)$.

method of calculation is similar to that of Miller and Sant, ${ }^{4}$ in which no sample was present, and to that of Goebel, ${ }^{5}$ in which the calculations were made for a sphere with a nonuniform coating.

With the equations for a single integrating sphere we calculate the effect of the multiple exchange of light between two adjacent spheres (Section 3). This gives the power detected in each sphere as a function of the reflectance and the transmittance of the sample. In addition we briefly consider the effects of a baffle between the sample and the detector (Section 4) and of a nonisotropic detector (Section 5).

\section{SINGLE SPHERES}

The signal given by a detector within an integrating sphere depends on the total power incident upon the detector and on the detector's response to that power. In Section 4 we consider the influence of a nonisotropic detector that responds to the light as a function of the angle at which the light is incident upon the detector. However, in this section and in Section 3 we assume that the detector collects light with the same efficiency over all the angles of incidence. Therefore the voltage given by the detector ouput will be directly proportional to the total light power incident upon the detector. The nomenclature used is summarized in Table 1.

\section{A. Light Incident upon the Sphere Wall}

Light enters the sphere and is incident upon the sphere wall with power $P$, as in Fig. 1A. The total power reflected (first reflection) is

$$
m P
$$

where $m$ is the coefficient of reflectance of the sphere wall. Since the wall is a Lambertian surface, the reflected power is distributed uniformly over the sphere wall. Therefore the power collected over a given area is proportional to the total power emitted $(m P)$ and the fractional area of the sphere that this area represents. Hence for the first reflection a detector of surface area $\delta$ will collect

$$
\frac{\delta}{A} m P
$$

where $A$ is the total inner surface area of the sphere, including the sphere wall, the detector, the sample, and the holes.

Similarly the total light from the first reflection collected by the walls of the sphere is

$$
\alpha m P \text {, }
$$

the total light collected by the sample is

$$
\frac{s}{A} m P,
$$

and the total light (lost through) the holes is

$$
\frac{h}{A} m P \text {, }
$$

where $\alpha=1-(\delta / A+s / A+h / A)$ is the area of the actual sphere wall relative to the surface area of the whole sphere $(A), s$ is the area of the sample, and $h$ is the sum of the areas of all the holes (ports) in the sphere.

Of the total power from the first reflection incident upon the detector [expression (2)], a fraction $r$, where $r$ is the coefficient of reflection for the detector, will be reflected. Similarly, for the wall [expression (3)] a fraction $m$ will be reflected, and for the sample [expression (4)] a fraction $R_{d}$ will be reflected ( $R_{d}$ is the coefficient of diffuse reflection of the sample). Thus the total reflected light of the second reflection will be

$$
r \frac{\delta}{A} m P+m \alpha m P+R_{d} \frac{s}{A} m P=m P F,
$$

where

$$
F=r \frac{\delta}{A}+m \alpha+R_{d} \frac{s}{A}
$$

represents the fraction of the total incident light that is diffusely reflected by all the components of the sphere. 
From this second reflection the power is distributed uniformly around the sphere so that the detector collects

$$
\frac{\delta}{A} m P F
$$

the wall collects

$$
\alpha m P F,
$$

and the sample collects

$$
\frac{s}{A} m P F
$$

In a similar manner the next (the third) reflection yields a power incident upon the detector of

$$
\frac{\delta}{A} m P F F
$$

and in general the detector collects from the $n$th reflection a power of

$$
\frac{\delta}{A} m P F^{n-1}
$$

Summing expression (12) for $n=1$ to $\infty$, we have the total power collected by the detector

$$
P_{d}=\frac{\delta}{A} m P\left(1+F+F^{2}+F^{3}+\ldots+F^{n-1}+\ldots\right) .
$$

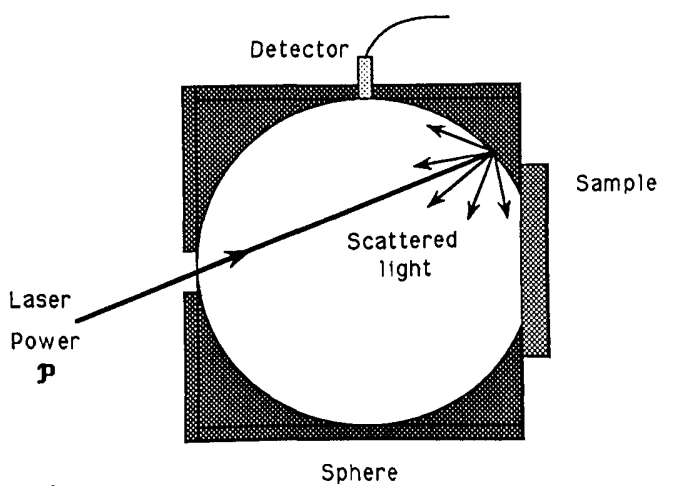

A

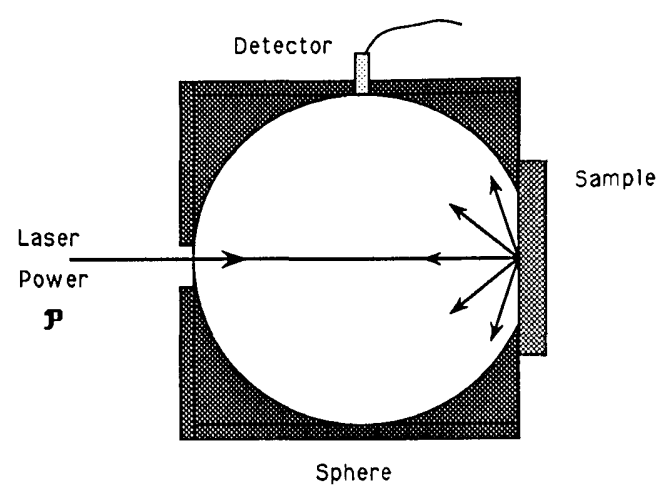

B

Fig. 1. Single-sphere geometries. Measurements of reflectance are made when the light enters through a port and is incident either upon the sphere wall (A) or directly upon the sample (B). In the latter case the light is incident perpendicularly to the sample, and therefore the specularly reflected light exits the sphere through the entrance port. Transmittance measurements may be made with either a diffuse (C) or a collimated (D) source incident upon the sample. In the latter case the light that is not scattered within the sample is then incident upon the far sphere wall, where it is diffusely reflected.

$$
\begin{aligned}
& \text { As } F<1 \\
& P_{d}=\frac{\delta}{A} \frac{m}{1-F} P=\frac{\delta}{A} \frac{m}{1-\left[m \alpha+R_{d}(s / A)+r(\delta / A]\right.} P .
\end{aligned}
$$

The equations of this section are summarized in Table 2. Note that, for any one reflection, energy is conserved. That is, the sum of the light reflected (columns 2 and 3) is equal to the sum of the light collected and lost (columns 4-7). A summation of the detected powers in column 7 yields Eq. (14). Since the source of the light incident upon the sample is diffuse, we refer to this case as diffuse incidence, as opposed to collimated incidence, in which the light is incident directly upon the sample.

Equation (14) is compatible with those of Miller and Sant $^{4}$ (no sample) and Goebel ${ }^{5}$ (general theory), which were calculated in a similar manner, and with those of Jacquez and Kuppenheim, ${ }^{2}$ who used an integral equation of the Fredholm type, and O'Brein, ${ }^{3}$ who used network representation.

\section{B. Light Incident upon the Sample}

Collimated light is incident upon the sample with power $P$, as in Fig. 1B. The power reflected consists of two parts, a collimated specular part,

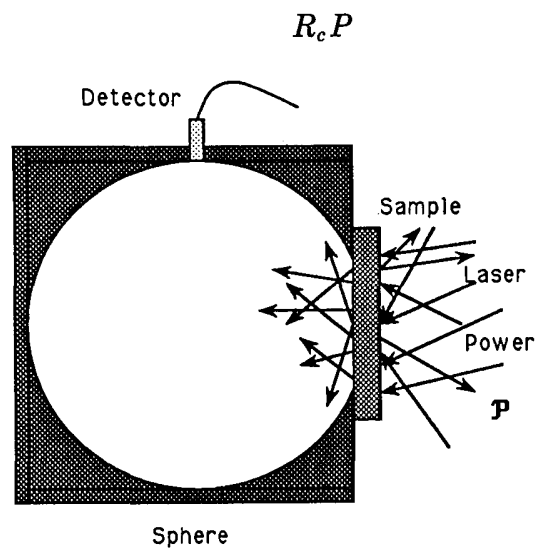

C

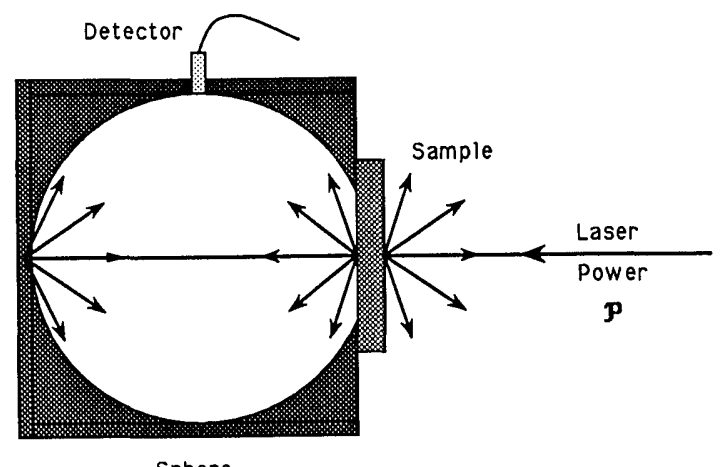

Sphere 


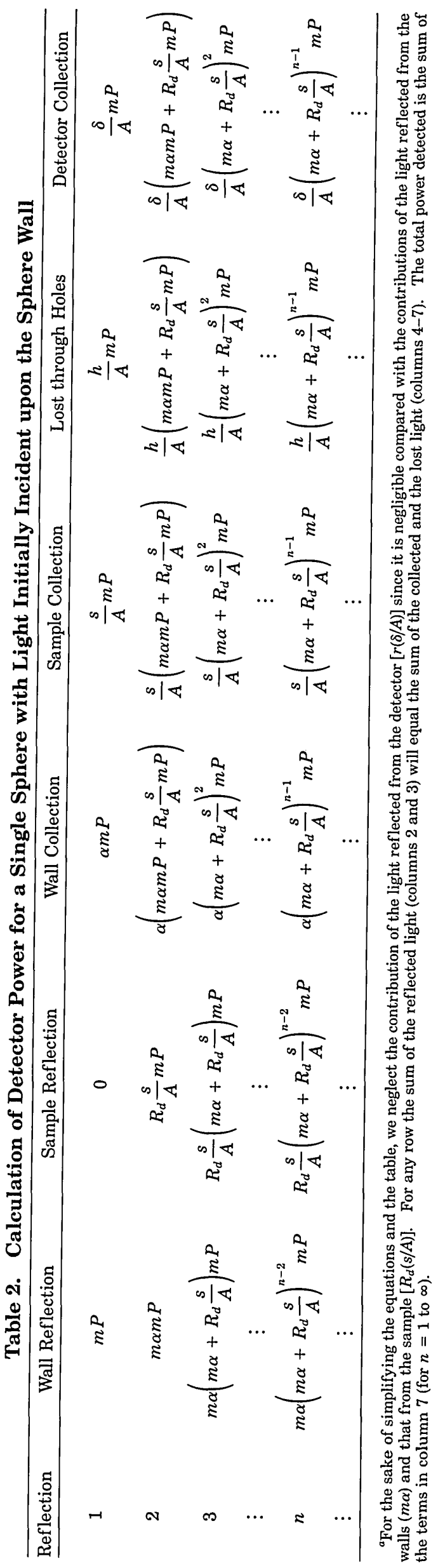


and a diffuse part

$$
R_{c d} P,
$$

where $R_{c}$ is the coefficient for the collimated (specular) reflection of collimated incident light and $R_{c d}$ is the coefficient for the diffuse reflection of collimated incident light.

If the collimated part [expression (15)] is incident upon the sphere wall (such as may be the case when the incoming light is not incident perpendicularly upon the sample), then there will be generation of diffuse light reflected from the sphere wall with a power of

$$
m R_{c} P \text {. }
$$

Therefore there are two diffuse sources of light [expressions (16) and (17)], each of which behaves in a manner similar to that of the diffuse source discussed in Subsection 2.A [expression (1)]. The power collected by the detector will be the sum of the multiply reflected light, as in Eq. (14), for each of the two sources. That is,

$$
P_{d}=\frac{\delta}{A} \frac{m R_{c}+R_{c d}}{1-\left[m \alpha+R_{d}(s / A)+r(\delta / A)\right]} P .
$$

This equation was verified by the process described in Subsection 2.A and in Table 2.

\section{Transmission}

To measure transmittance we place the sample at the entrance port of a sphere, as in Figs. 1C and 1D. The sample may be illuminated with either diffuse or collimated light of power $P$.

Once again the problem reduces to that of one or two diffuse sources, the first being the light from the sample. This source is for diffuse light incident upon the sample,

$$
T_{d} P,
$$

or for collimated light incident upon the sample,

$$
T_{c d} P,
$$

where $T_{d}$ and $T_{c d}$ are the diffuse transmission coefficients for diffuse and collimated incident light, respectively.

If the light incident upon the sample is collimated and the collimated transmitted light does not leave the sphere through a hole, then there is a second source of diffuse light where the collimated transmitted light strikes the sphere (Fig. 1D),

$$
m^{\prime} T_{c} P
$$

where $m^{\prime}$ is the coefficient of diffuse reflection from the sphere wall. We introduce the prime notation here since we will use it below to distinguish between the sphere collecting primarily transmitted light (prime, transmittance sphere) and that collecting primarily reflected light (no prime, reflectance sphere).

Therefore, through the same procedure as in Subsection 2.A, we obtain the total power collected by the detector for diffuse light incident upon the sample,

$$
P_{d}^{\prime}=\frac{\delta^{\prime}}{A^{\prime}} \frac{T_{d}}{1-\left[m^{\prime} \alpha^{\prime}+R_{d}\left(s / A^{\prime}\right)+r^{\prime}\left(\delta^{\prime} / A^{\prime}\right)\right]} P ;
$$

for collimated light incident upon the sample, the detected power is

$$
P_{d}^{\prime}=\frac{\delta^{\prime}}{A^{\prime}} \frac{T_{c d}+m^{\prime} T_{c}}{1-\left[m^{\prime} \alpha^{\prime}+R_{d}\left(s / A^{\prime}\right)+r^{\prime}\left(\delta^{\prime} / A^{\prime}\right)\right]} P
$$

\section{DOUBLE SPHERES}

The simultaneous measurement of the reflectance and the transmittance of the sample requires that the sample be placed between two spheres, as in Fig. 2. The sample is then at the exit port of the first sphere measuring reflectance (referred to as the reflectance sphere) and at the entrance port of the second sphere measuring transmittance (referred to as the transmittance sphere). We require only that these two ports be identical in size. If they are not, some of the light transmitted by the sample will not be collected within the spheres. The spheres may have different wall, hole, and detector areas and different wall reflectances. We assume that the transmittance and the reflectance of light are homogeneous with respect to which side of the sample the light is incident upon.
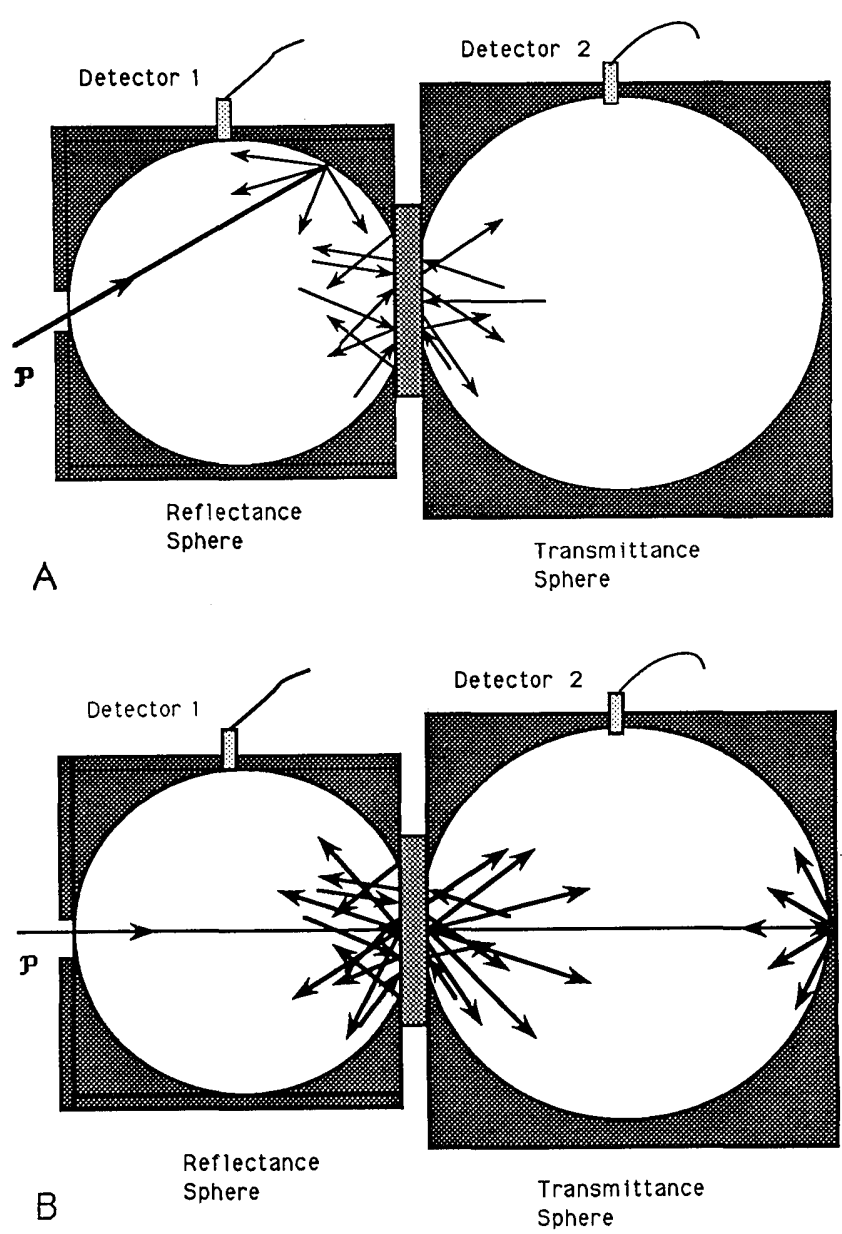

Fig. 2. Double-sphere geometries. Light may enter the system through the entrance port of the reflectance sphere and be incident upon the wall $(\mathrm{A})$, the so-called diffuse case, or upon the sample (B), the collimated case. There is a multiple exchange of light between the spheres, and in the collimated case some of the light transmitted through the sample without scattering may be incident upon the far sphere wall, where it is diffusely reflected. 


\section{A. Diffuse Light}

Consider light incident upon the side of the wall of the reflectance sphere (Fig. 2A) as in Subsection 2.A and rewrite Eq. (14) as

$$
P_{d 1}=\frac{\delta}{A} \frac{m}{V} P,
$$

where

$$
V=1-\left(m \alpha+R_{d} \frac{s}{A}+r \frac{\delta}{A}\right)
$$

and where the subscript 1 has been added to $P_{d}$ to indicate that this power is only part of the total power detected. It is the power incident upon the detector surface within the reflectance sphere that is due only to the light generated within the reflectance sphere that has not traveled through the sample from the transmittance sphere.

Since $P_{d 1}$ is the power incident upon the detector of surface area $\delta$, we may deduce that

$$
P_{s 1}=\frac{s}{A} \frac{m}{V} P
$$

is the power incident upon the sample.

We know that this power $\left(P_{s 1}\right)$ multiplied by the coefficient for the diffuse transmission of diffuse incident light $\left(T_{d}\right)$ will provide a source for the transmittance sphere, as in expression (19), such that there is a power incident upon the detector in the transmittance sphere of

$$
\begin{aligned}
P_{d 1}{ }^{\prime} & =\frac{\delta^{\prime}}{A^{\prime}} \frac{1}{V^{\prime}} T_{d} P_{s 1} \\
& =\frac{\delta^{\prime}}{A^{\prime}} \frac{T_{d}}{V^{\prime}} \frac{s}{A} \frac{m}{V} P,
\end{aligned}
$$

where $V^{\prime}$ is identical to $V$ in nomenclature except that it and the other appropriate sphere parameters have been changed (prime added) to account for the possibility of the transmittance sphere's being different from the reflectance sphere.

A fraction of light transmitted into the transmittance sphere is incident back upon the sample,

$$
P_{s 1}^{\prime}=\frac{s}{A^{\prime}} \frac{1}{V^{\prime}} T_{d} P_{s 1},
$$

and acts as a second source for the reflectance sphere $\left(P_{s 1}{ }^{\prime} T_{d}\right)$ such that the detector detects an additional power of

$$
\begin{aligned}
P_{d 2} & =\frac{\delta}{A} \frac{1}{V} T_{d} P_{s 1}{ }^{\prime} \\
& =\frac{\delta}{A} \frac{m}{V} \frac{T_{d}}{V} \frac{T_{d}}{V^{\prime}} P .
\end{aligned}
$$

Similarly some of this second source is incident back upon the sample and is transmitted. This light acts as a second source in the transmittance sphere.

This process of the exchange of light between the two spheres continues ad infinitum, and the total light incident upon the detectors may be calculated as a sum of a geometric series of the power detected for each source (each exchange of light) within each sphere to give, for the reflectance sphere, a total detected power of

$$
P_{d}=\frac{\delta}{A} \frac{m}{V} \frac{1}{1-T T^{\prime}} P
$$

and, for the transmittance sphere, a total detected power of

$$
P_{d}^{\prime}=\frac{\delta^{\prime}}{A^{\prime}} \frac{s}{A} \frac{m T_{d}}{V} \frac{1}{V^{\prime}} \frac{1}{1-T T^{\prime}} P,
$$

where

$$
T=\frac{s}{A} \frac{T_{d}}{V}
$$

and

$$
T^{\prime}=\frac{s}{A^{\prime}} \frac{T_{d}}{V^{\prime}} .
$$

The calculations described here are summarized in Table 3, where Eqs. (30) and (31) may be calculated by summing columns 3 and 5 , respectively.

\section{B. Collimated Light}

If collimated light is first incident upon the sample within the reflectance sphere, then we may calculate the power detected in each sphere by using procedure identical to that in Subsection 3.A but now with two initial sources in the reflectance sphere [expressions (16) and (17)] and two in the transmittance sphere [expressions (20) and (21)]. Combining these expressions with Eqs. (30) and (31) yields the following results. be

For the reflectance sphere the total detected power will

$$
P_{d}=\frac{\delta}{A} \frac{\left[R_{c d}+m R_{c}+T^{\prime}\left(T_{c d}+m^{\prime} T_{c}\right)\right]}{V\left(1-T T^{\prime}\right)} P,
$$

and for the transmittance sphere the total detected power will be

$$
P_{d}^{\prime}=\frac{\delta^{\prime}}{A^{\prime}} \frac{\left[T_{c d}+m T_{c}+T\left(R_{c d}+m R_{c}\right)\right]}{V^{\prime}\left(1-T T^{\prime}\right)} P .
$$

\section{Discussion of the Sphere Equations}

The equations for the power detected in the double-sphere system [Eqs. (30), (31), (34), and (35)] may be understood in terms of the equations for the single-sphere systems [Eqs. (14), (18), (22), and (23)] and in terms of the sources of diffuse light.

To explain the equations, we use the example of a collimated source. In the single- and the double-sphere cases for reflected light there are two diffuse-light sources,

$$
\begin{gathered}
R_{c d} P, \\
m R_{\mathrm{c}} P .
\end{gathered}
$$

In the double-sphere case there is a third source of diffuse light that is due to a proportion of the collimated light first incident upon the sample that is transmitted through the sample into the transmittance sphere. Some of this 


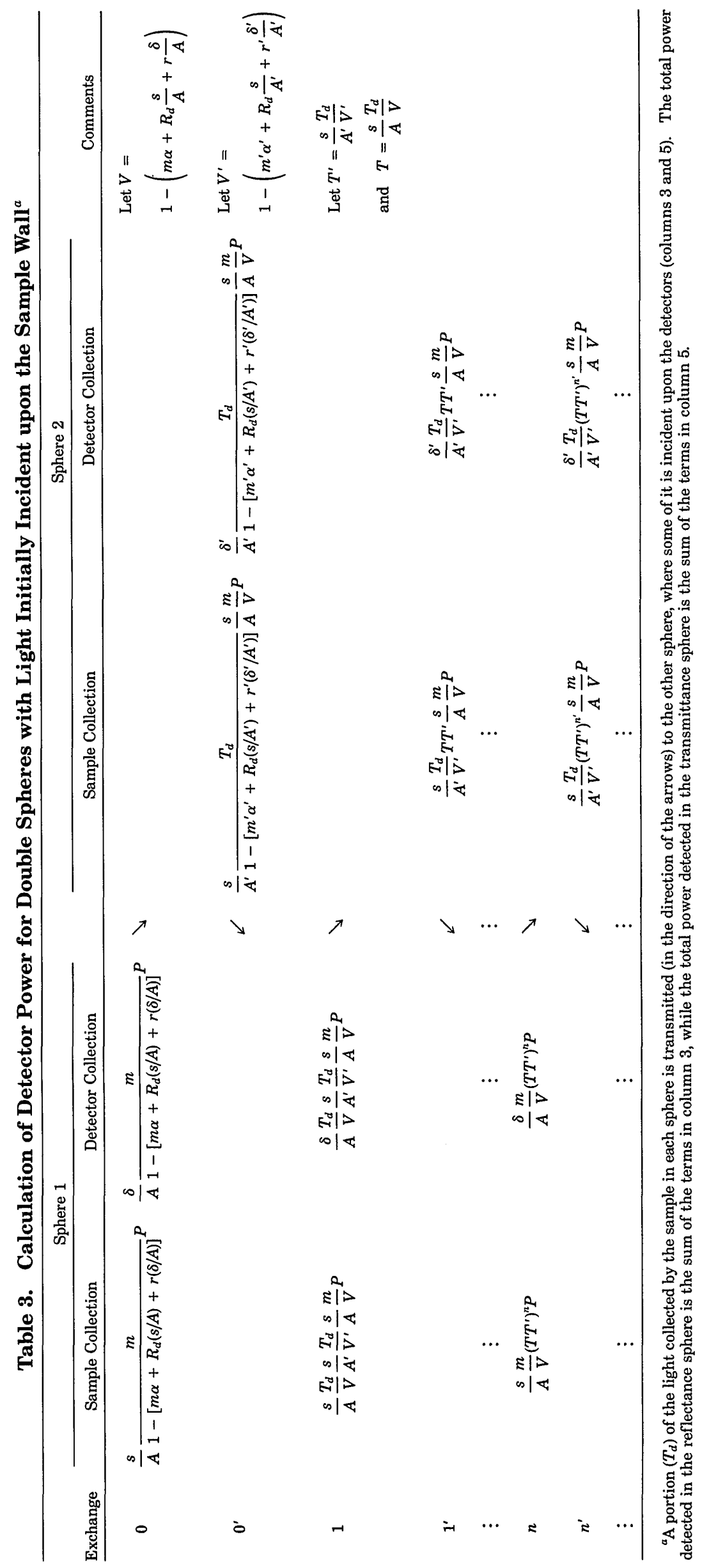


light is again transmitted through the sample, this time back into the reflectance sphere to act as the third diffuse source:

$$
\frac{s}{A^{\prime}} \frac{T_{d}\left(T_{c d}+m^{\prime} T_{c}\right)}{V^{\prime}} P=T^{\prime}\left(T_{c d}+m^{\prime} T_{c}\right) P .
$$

Within the reflectance sphere these three diffuse sources will undergo multiple reflections, and some light will be lost to absorption by the wall, through the holes, and to absorption and transmission by the sample, in the same fashion as was discussed in Subsection 2.A. The power detected in the reflectance sphere is then

$$
P_{d}=\frac{\delta}{A} \frac{R_{c d}+m R_{c}+T^{\prime}\left(T_{c d}+m^{\prime} T_{c}\right)}{V} P .
$$

However, the presence of the transmittance sphere means that some of the light incident upon the sample will be transmitted into the transmittance sphere, and some of this light will then be transmitted back into the reflectance sphere. The factor $1 /\left(1-T T^{\prime}\right)$ in Eq. (35) accounts for the increase in the detected power that is due to this multiple exchange of light between the spheres.

Similarly in the transmittance sphere there are three diffuse sources:

$$
\begin{gathered}
T_{c d} P, \\
m^{\prime} T_{c} P, \\
\frac{s}{A} \frac{T_{d}\left(R_{c d}+m R_{c}\right)}{V} P=T\left(T_{c d}+m T_{c}\right) P .
\end{gathered}
$$

The first two sources arise from the transmission of some of the collimated light directly incident upon the sample, and the third arises from the transmission of some of the diffuse light generated within the reflectance sphere. Once again the light is attenuated by losses within the sphere proportional to $1 / V^{\prime}$, and the signal is increased by the factor $1 /\left(1-T T^{\prime}\right)$ because of the multiple exchange of light between the spheres.

\section{INFLUENCE OF A BAFFLE}

Often within integrating spheres a baffle is placed between the sample and the detector to avoid the collection of specularly reflected light from the sample. If we assume that the baffle is small and acts as a diffuse reflector placed in the sphere wall at the position of the detector with the same reflectance as the sphere wall, then the light emitted from the sample in the direction of the detector, less that lost through absorption by the baffle, will be returned to the sphere. Therefore we neglect the term $r \delta / A$ that is due to the reflection of light from the detector. This is reasonable since this reflection is small compared with the reflections by the wall and the sample.

For diffuse incident light the calculations proceed in the same manner as in Subsection 2.A except that expression (8), the power detected from the second reflection, becomes

$$
\frac{\delta}{A} m \alpha m P,
$$

which is a factor $\delta / A\left[R_{d}(s / A) m P\right]$ less than expression (8) owing to the detector's not collecting the light directly reflected from the sample. As a further consequence, expression (11), the power detected from the third reflection, becomes

$$
\frac{\delta}{A} m \alpha m P\left(m \alpha+R_{d} \frac{s}{A}\right)
$$

and expression (12), the power detected from the $n$th reflection, becomes

$$
\frac{\delta}{A} m \alpha m P\left(m \alpha+R_{d} \frac{s}{A}\right)^{n-2}
$$

Finally the equation for the total detected power reduces to

$$
P_{d}=\frac{\delta}{A} \frac{\left[1-R_{d}(s / A)\right] m}{1-\left[m \alpha+R_{d}(s / A)\right]} P
$$

For collimated incident light a similar argument applies to the first diffuse source $\left(R_{c d} P\right)$, taking into account the nondetection of light from the first reflection. The contribution from the second diffuse source $\left(m R_{c}\right)$ is calculated in a manner identical to that used above. For double spheres, a similar argument applies, with the exchange of light between the spheres being identical to that discussed in Section 3. Table 4 summarizes the equations for single and double spheres with and without baffles.

\section{INFLUENCE OF A NONISOTROPIC DETECTOR}

An alternative to a baffle is a nonisotropic detector, in particular, a detector that sees only the opposite sphere wall. This is the case in Fig. 3, in which the detector sees a fractional area $\beta$ of the sphere wall and absorbs light with an efficiency $E(\theta, \phi)$, where $\theta$ and $\phi$ are the two defining angles of any direction in the incoming hemisphere and $E<1 \forall \theta, \phi . \quad E$ is zero at $\theta$ and $\phi$ where the light does not emanate from the wall area defined by $\beta$.

Since the area $\beta$ does not contain the sample, the analysis proceeds in a manner identical to that of the analysis for a baffle, except that the detector now detects only a fraction of the light incident upon it, this fraction being

$$
f=\beta \int_{\pi / 2} \int_{2 \pi} E(\theta, \phi) \sin \theta \mathrm{d} \theta \mathrm{d} \phi .
$$

The power detected will also depend on the point of incidence of the collimated light. Thus for diffuse light Eq. (46) becomes

$$
P_{d}=\frac{\delta}{A}\left\{E^{*} m+\frac{f m \alpha m}{1-\left[m \alpha+R_{d}(s / A)+r(\delta / A)\right]}\right\} P,
$$

where

$$
E^{*}=\left\{\begin{array}{ll}
0 & \text { if point of incidence } \notin \beta \\
E\left(0^{*}, \phi^{*}\right) & \left(\theta^{*}, \phi^{*}\right) \text { define the point of incidence } \\
& \text { relative to the detector }
\end{array} .\right.
$$

Once again, similar equations may be formed for the collimated incidence, the transmitted light, and the double integrating spheres. 
Table 4. Summary of Detector Power $^{a}$

\begin{tabular}{|c|c|c|}
\hline Geometry & No Baffle & Baffle \\
\hline \multicolumn{3}{|l|}{ Single spheres } \\
\hline \multicolumn{3}{|l|}{ Diffuse incident light } \\
\hline \multirow{2}{*}{ Reflectance } & $\frac{\delta}{m} P$ & $\frac{\delta}{m\left[1-R_{d}(s / A)\right]} P$ \\
\hline & $\left.\bar{A} \overline{1-\left[m \alpha+R_{d}(s / A)+r(\delta / A)\right]}\right]^{\mathrm{T}}$ & $\bar{A} 1-\left[m \alpha+R_{d}(s / A)\right]^{2}$ \\
\hline \multicolumn{3}{|l|}{ Collimated incident light } \\
\hline \multirow{2}{*}{ Reflectance } & $\underline{\delta}-m R_{\mathrm{c}}+R_{c d}$ & $\underline{\delta} \underline{m R_{c}\left[1-R_{d}(s / A)\right]+R_{c d} m \alpha} P$ \\
\hline & $\bar{A} \overline{1-\left[m \alpha+R_{d}(s / A)+r(\delta / A)\right]}$ & $\bar{A} \quad 1-\left[m \alpha+R_{d}(s / A)\right]$ \\
\hline \multicolumn{3}{|l|}{ Diffuse incident light } \\
\hline \multirow{2}{*}{ Transmittance } & $\underline{\delta^{\prime}}$ & $\underline{\delta^{\prime}}$ \\
\hline & $A^{\prime} 1-\left[m^{\prime} \alpha^{\prime}+R_{d}\left(s / A^{\prime}\right)+r^{\prime}\left(\delta^{\prime} / A^{\prime}\right)\right]^{1}$ & $A^{\prime} 1-\left[m^{\prime} \alpha^{\prime}+R_{d}\left(s / A^{\prime}\right)\right]^{\alpha}$ \\
\hline \multicolumn{3}{|l|}{ Collimated incident light } \\
\hline \multirow{2}{*}{ Transmittance } & $\underline{\delta}^{\prime}$ & $\underline{\delta}^{\prime} \frac{m^{\prime} T_{c}\left[1-R_{d}\left(s / A^{\prime}\right)\right]+T_{c d} m^{\prime} \alpha^{\prime}}{P}$ \\
\hline & $\left.\overline{A^{\prime}} \overline{1-\left[m^{\prime} \alpha^{\prime}+R_{d}\left(s / A^{\prime}\right)+r^{\prime}\left(\delta^{\prime} / A^{\prime}\right)\right]}\right]^{r}$ & $A^{\prime} \quad 1-\left[m^{\prime} \alpha^{\prime}+R_{d}\left(s / A^{\prime}\right)\right]$ \\
\hline \multicolumn{3}{|l|}{ Double spheres } \\
\hline \multicolumn{3}{|l|}{ Diffuse incident light } \\
\hline \multirow{2}{*}{ Reflectance sphere } & $\frac{\delta}{m}-\frac{1}{-} P$ & $\underline{\delta} \stackrel{m\left[1-R_{d}(s / A)\right]}{-1}-P$ \\
\hline & $A 1-\left[m \alpha+R_{d}(s / A)+r(\delta / A)\right] 1-T T^{\prime}$ & A $1-\left[m \alpha+R_{d}(s / A)\right] 1-T T^{\prime}$ \\
\hline \multirow{4}{*}{ Transmittance sphere } & $\underline{\delta}^{\prime} s-m T_{d}$ & $\underline{\delta}^{\prime} \underline{s} \underline{m T_{d} m^{\prime} \alpha^{\prime}}$ \\
\hline & $\overline{A^{\prime}} \bar{A} \overline{1-\left[m \alpha+R_{d}(s / A)+r(\delta / A)\right]}$ & $\overline{A^{\prime}} \bar{A} \overline{1-\left[m \alpha+R_{d}(s / A)\right]}$ \\
\hline & $\times \frac{1}{1} P$ & $\times \frac{1}{1}-\frac{1}{}$ \\
\hline & $1-\left[m^{\prime} \alpha^{\prime}+R_{d}\left(s / A^{\prime}\right)+r^{\prime}\left(\delta^{\prime} / A^{\prime}\right)\right] 1-T T^{\prime}$ & $1-\left[m^{\prime} \alpha^{\prime}+R_{d}\left(s / A^{\prime}\right)\right] 1-T T^{\prime}$ \\
\hline \multicolumn{3}{|l|}{ Collimated incident light } \\
\hline \multirow{2}{*}{ Reflectance sphere } & $\underline{\delta} \quad m R_{c}+R_{c d}+T^{\prime}\left(T_{c d}+m^{\prime} T_{c}\right)$ & $\underline{\delta} \underline{m R_{c}\left[1-R_{d}(s / A)\right]+R_{c d} m \alpha+T^{\prime}\left(T_{c d}+m^{\prime} T_{c}\right) m \alpha} P$ \\
\hline & $A\left\{1-\left[m \alpha+R_{d}(s / A)+r(\delta / A)\right]\right\}\left(1-T T^{\prime}\right)^{2}$ & $A \quad\left\{1-\left[m \alpha+R_{d}(s / A)\right]\right\}\left(1-T T^{\prime}\right)$ \\
\hline \multirow{2}{*}{ Transmittance sphere } & $\underline{\delta}^{\prime} \underline{m^{\prime} T_{c}+T_{c d}+T\left(R_{c d}+m R_{c}\right)} P$ & $\underline{\delta^{\prime}} \underline{m^{\prime} T_{c}\left[1-R_{d}\left(s / A^{\prime}\right)\right]+T_{c d} m^{\prime} \alpha^{\prime}+T\left(R_{c d}+m R_{c}\right) m^{\prime} \alpha^{\prime}} P$ \\
\hline & $A^{\prime}\left\{1-\left[m^{\prime} \alpha^{\prime}+R_{d}\left(s / A^{\prime}\right)+r^{\prime}\left(\delta^{\prime} / A^{\prime}\right)\right]\right\}\left(1-T T^{\prime}\right){ }^{1}$ & $A^{\prime} \quad\left\{1-\left[m^{\prime} \alpha^{\prime}+R_{d}\left(s / A^{\prime}\right)\right]\right\}\left(1-T T^{\prime}\right)$ \\
\hline
\end{tabular}

${ }^{a}$ For the sake of simplicity the equations for the baffle neglect the small contribution to the power of the sphere that is due to the reflected light from the detector since this light $[r(\delta / A)]$ is negligible relative to the light reflected by the wall or sample. Also note that $T=(s / A)\left\{T_{d} / 1-\left[m \alpha+R_{d}(s / A)+r(\delta / A)\right]\right\}$ and $T^{\prime}=\left(s / A^{\prime}\right)\left\{T_{d} / 1-\left[m^{\prime} \alpha^{\prime}+R_{d}\left(s / A^{\prime}\right)+r^{\prime}\left(\delta^{\prime} / A^{\prime}\right)\right]\right\}$.

\section{DISCUSSION}

The equations calculated for the power measured with the double integrating spheres are required for the simultaneous measurement of the reflection and the transmission properties of the sample. With these properties a suitable model of radiative transfer within the sample may be applied to deduce the absorption and the scattering characteristics of the sample. Such calculations and simultaneous measurements are particularly relevant to the field of laser surgery since they permit measurements to be made during the heating of the tissue and ultimately permit more precise dosimetry. This is by no means the only application of the double integrating sphere; for example, measurements of materials under cooling, under pressure, and during chemical change may be made.

The power measured in each sphere is a product of the sum of the diffuse sources within the sphere with a function (greater than 1) that accounts for the multiple reflections within that sphere and, in the case of the double spheres, a function (also greater than 1) that accounts for an increase in the detected intensity that is due to the multiple exchange of light between the spheres.

The first function for multiple reflections within the sphere $\left\{1 /\left[1-\left(m \alpha+R_{d} s / A+r \delta / A\right)\right]\right\}$ takes into account the losses within the sphere resulting from absorption by the sphere wall and the sample and from light's exiting the sphere through holes. This function is related to the sphere efficiency ${ }^{5}$ since it describes the deviation from a perfect sphere in which there are no light losses.

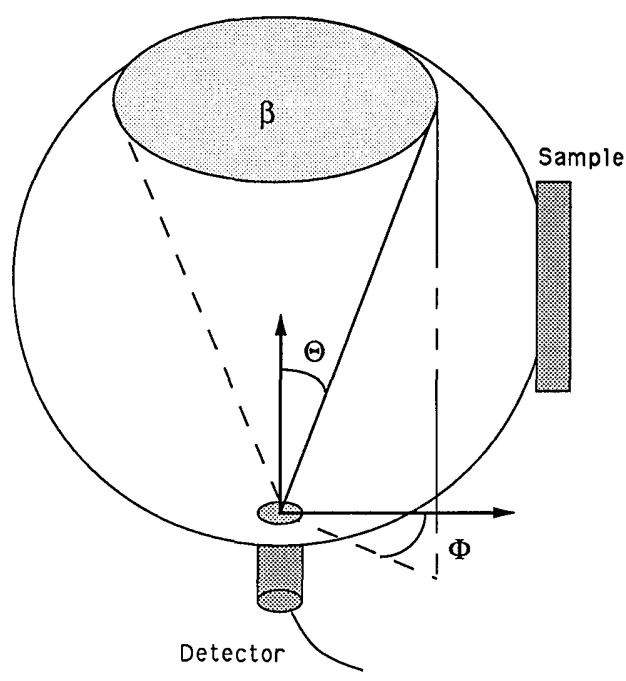

Fig. 3. Nonisotropic detector in the sphere wall sees only the light that is reflected from the portion of the sphere wall marked $\beta$. Furthermore, the detector efficiency may vary as to the direction $(\theta, \phi)$ of the incoming light from $\beta$. 


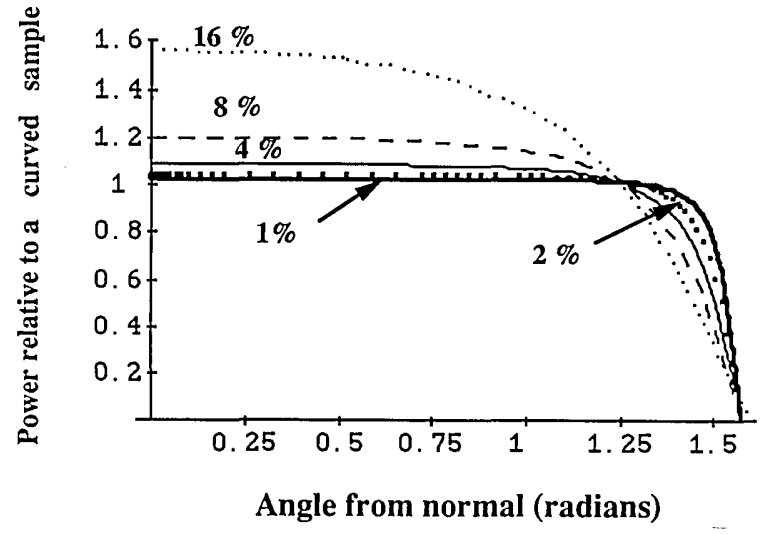

Fig. 4. In the case of a flat sample, as opposed to a curved sample that fits the circumference of the sphere wall, the power collected within the sphere from a diffusely emitting element depends on the angle between the emittor and the collecting portion of the sphere (angle from normal) and on the sample area relative to the total sphere surface area (1-16\%).

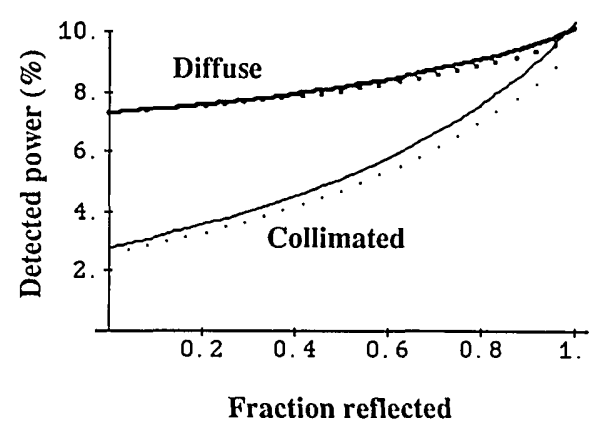

(a)

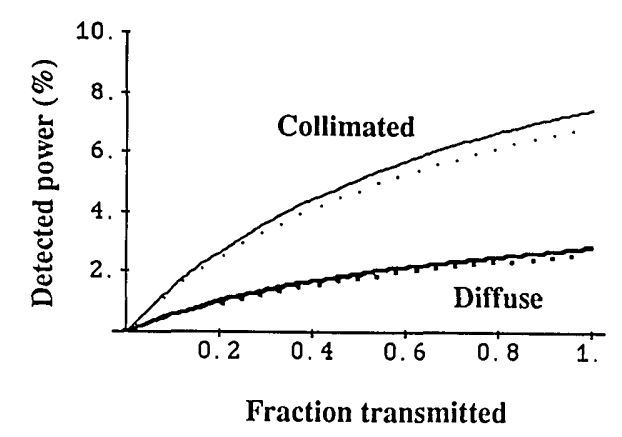

(b)

Fig. 5. Percentage of detected power in (a) the reflectance sphere and (b) the transmittance sphere relative to the total incident power (for the double-sphere geometry). Shown are the powers for diffuse light collected without a baffle (solid thick curves) and with a baffle (dotted thick curves) and for collimated light without a baffle (solid thin curves) and with a baffle (dotted thin curves). In these examples the sphere-wall reflectance $(m)$ is 0.98 , the relative sample area $(s / A)$ is 0.03 , the relative hole area $(h / A)$ is 0.025 , and the relative detector area $(\delta / A)$ is 0.005 . There is assumed to be no collimated reflectance or transmittance $\left(R_{c}=T_{c}=0\right)$, and $R_{c d}=R_{d}, T_{c d}=T_{d}=1-R_{d}$.

The calculations presented in this paper have assumed that the sample area is small compared with the sphere area and that the sample is curved to fit the sphere surface. However, in practice most samples are flat, and therefore the power diffusely reflected from them can no longer be considered to be evenly distributed over the sphere wall. Figure 4 illustrates the influence of a flat sample on the distribution of power on the sphere wall as a function of the angle from normal. For small areas (less than $4 \%$ ) this influence may often be neglected. Jacquez and Kuppenheim ${ }^{2}$ considered the flat sample in detail for the single integrating sphere.

For two communicating spheres the function describing the exchange of light between the spheres $\left[1 /\left(1-T T^{\prime}\right)\right]$ depends on the efficiency of each sphere, on the sample size, and on the transmittance of the sample. For smaller samples (relative to the total sphere area), samples that transmit less, and less-efficient spheres (greater light losses), the exchange of light between the spheres is reduced.

The differences in the detected power for collimated and diffuse incident light and for spheres with and without baffles are illustrated in Fig. 5. In this example the sample is assumed to be nonabsorbing, and there is assumed to be no collimated transmitted light and no specular reflectance. That is, $T_{c}=R_{c}=0, R_{c d}=R_{d}$, and $T_{c d}=$ $T_{d}=1-R_{d}$. Figure 5 assumes identical spheres with a sphere-wall reflectance $(m)$ of 0.98 , a relative sample area $(s / A)$ of 0.03 , a relative hole area $(h / A)$ of 0.025 , and a relative detector area $(\delta / A)$ of 0.005 . There is always a greater change in the collimated signal than in the diffuse signal for a small change in the reflectance or the transmittance. This suggests that collimated light is likely to

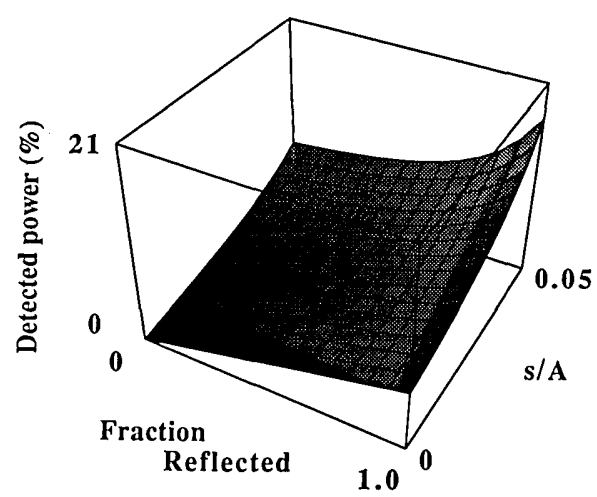

(a)

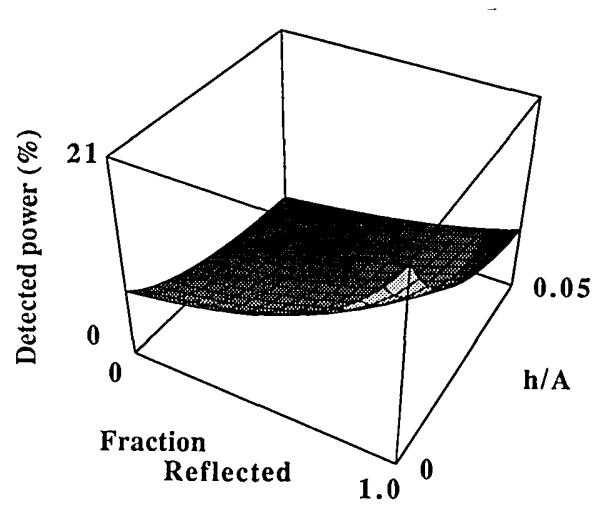

(b)

Fig. 6. Under the same conditions as in Fig. 5, the percentage of detected power in the reflectance sphere (double-sphere geometry) as a function of the sample reflectance and as a function of (a) the relative sample area $(s / A)$ for a constant relative hole area $(h / A)$ of 0.025 or (b) the relative hole area $(h / A)$ for a constant relative sample area $(s / A)$ of 0.03 . 
make a more accurate measurement of reflectance and/or transmittance. For 0 reflectance there is a signal in the reflectance sphere even for collimated light. This is because the sphere receives some light from the transmittance sphere. For the example given this signal is a maximum because all the light is assumed to be transmitted (diffusely). For a sample that absorbs or that is less scattering, this 0 reflectance signal will be relatively smaller. To illustrate the effect of changing the sample size or the size of the holes, Figs. 6(a) and 6(b) show the signal in the reflectance sphere as a function of the reflectance for a relative sample area of 0 to 0.05 for a constant relative hole area (0.025) and for a relative hole area of 0 to 0.05 for a constant relative sample area (0.03), respectively.

Overall, six coefficients of sample reflectance and transmittance have been presented. Six measurements must be made to deduce all these parameters. The diffuse reflectance $\left(R_{d}\right)$ and transmittance $\left(T_{d}\right)$ of diffuse incident light may be calculated from the power measured in the two spheres [Eqs. (30) and (31), respectively] when the light is initially incident upon the wall of the reflectance sphere. These values and the power measured in each of the spheres for collimated incident light [Eqs. (34) and (35)] will yield the diffuse reflectance $\left(R_{c d}\right)$ and transmittance $\left(T_{c d}\right)$ of collimated incident light if the specular reflectance and the collimated transmitted light are allowed to leave the sphere system. The measurement of the power of the collimated specular reflectance and the collimated transmitted light by two other detectors outside the spheres will yield $R_{c}$ and $T_{c}$.

All six measurements need not be made if only certain information is required. For example, for the calculation of radiative-transfer-theory scattering and absorption coefficients and the average of the cosine of the scattering angle, only three light measurements (diffuse reflectance and diffuse and collimated transmittance) are required. An appropriate radiative transfer $\operatorname{model}^{9}$ will relate the coefficient of diffuse reflectance for diffuse incident light $\left(R_{d}\right)$ with the coefficient for collimated incident light $\left(R_{c d}\right)$ and similarly will relate $T_{d}$ and $T_{c d}$.

\section{ACKNOWLEDGMENTS}

These investigations are in the program of the Foundation for Fundamental Research on Matter (FOM) and have been supported by Netherlands Technology Foundation (STW) grant VNS88.1426.

*Present address: Hogeschool, 's Hertogenbosch, The Netherlands.

†Present address: Wellman Laboratories of Photomedicine, Massachusetts General Hospital, Boston, Massachusetts 02114 .

\section{REFERENCES}

1. A. H. Taylor, "The measurement of diffuse reflection factors and a new absolute reflectometer," J. Opt. Soc. Am. 4, 9-23 (1920).

2. J. A. Jacquez and H. F. Kuppenheim, "Theory of the integrating sphere," J. Opt. Soc. Am. 45, 460-470 (1955).

3. P. F. O'Brien, "Network representation of the integrating sphere," J. Opt. Soc. Am. 46, 343-345 (1956).

4. O.E. Miller and A. J. Sant, "Incomplete integrating sphere," J. Opt. Soc. Am. 48, 828-831 (1958).

5. D. G. Goebel, "Generalized integrating-sphere theory," Appl. Opt. 6, 125-128 (1967).

6. S. L. Jacques, C. A. Alter, and S. A. Prahl, "Angular dependence of He-Ne laser light scattering by human dermis," Lasers Life Sci. 4, 309-333 (1987).

7. B. C. Wilson, M. S. Patterson, and S. T. Flock, "Indirect versus direct techniques for the measurement of the optical properties of tissues," Photochem. Photobiol. 46, 601-608 (1987).

8. H. C. van de Hulst, Multiple Light Scattering (Academic, New York, 1980), Vol. 1.

9. S. A. Prahl, "Light transport in tissue," $\mathrm{Ph} . \mathrm{D}$. dissertation (University of Texas at Austin, Austin, Texas, 1988).

10. M. Keijzer, S. L. Jacques, S. A. Prahl, and A. J. Welch, "Light distributions in tissue: Monte Carlo simulations for finitediameter laser beams," Lasers Surg. Med. 9, 148-154 (1989).

11. G. J. Derbyshire, D. K. Bogen, and M. Unger, "Thermally induced optical property changes in myocardium at $1.06 \mu \mathrm{m}$," Lasers Surg. Med. 10, 28-34 (1990).

12. P. Rol, P. Nieder, U. Dürr, P.-D. Henchoz, and F. Fankhauser, "Experimental investigations on the light scattering properties of the human sclera," Laser Light Ophthalmol. 3, 201212 (1990). 